\title{
Laparoscopic management of a pelvic retroperitoneal leiomyoma-case report
}

\author{
William Kondo • Revaz Botchorishvili • \\ Fanny Desvignes • Gérard Mage
}

Received: 1 August 2009 / Accepted: 15 October 2009/Published online: 7 November 2009

(C) Springer-Verlag 2009

\begin{abstract}
Leiomyomas are benign tumors frequently found in the fourth and fifth decades of life. Although the uterus is the most common site of origin of leiomyomas, they can develop at any site where there is smooth muscle cell. Extrauterine leiomyomas are not common and usually their diagnostic are more challenging. In this paper, we report one case of pelvic retroperitoneal leiomyoma associated to vulvar/perineal leiomyomas. A 47-year-old female patient presented with a 6-month history of deep dyspareunia, abdominal pain, dysuria, and pain during defecation. She had a previous history of two open myomectomies, a supracervical hysterectomy associated to the exeresis of a vulvar leiomyoma, and a left salpingo-oophorectomy. On vaginal examination, there was a tender and firm mass at the vaginal vault and along the posterior vaginal wall. There was another tumor at the left vulvar/perineal region measuring around $6 \mathrm{~cm}$. Magnetic resonance imaging demonstrated the presence of a mass at the Douglas pouch measuring $14 \times 10 \times 10 \mathrm{~cm}$ suggestive of uterine leiomyomatosis. She underwent a successful laparoscopic resection of the pelvic tumor with an operative time of $210 \mathrm{~min}$. The vulvar/perineal lesions were resected by vaginal approach. She presented an uneventful postoperative course and was discharged home 3 days after surgery. Histopathology confirmed the diagnosis of leiomyomas. Laparoscopic
\end{abstract}

\footnotetext{
W. Kondo · R. Botchorishvili · F. Desvignes · G. Mage

C.H.U. Clermont-Ferrand,

Department of Gynecology and Obstetrics,

Polyclinique de l'Hôtel Dieu,

Clermont Ferrand, France

W. Kondo $(\bowtie)$

Boulevard Leon Malfreyt,

63058 Clermont-Ferrand CEDEX 1, France

e-mail: williamkondo@yahoo.com

URL: drwilliamkondo.site.med.br
}

approach for pelvic retroperitoneal leiomyoma is feasible. It is important to keep in mind the possibility of this entity as a differential diagnosis of retroperitoneal masses in women.

Keywords Retroperitoneal leiomyomatosis · Leiomyoma · Retroperitoneal mass $\cdot$ Laparoscopy

\section{Introduction}

Leiomyomas are benign tumors, which are mainly composed of smooth muscle and some fibrous connective tissue elements [1]. They represent the most common gynecologic and uterine neoplasms [2], and $20 \%$ to $30 \%$ of women older than 35 years have uterine leiomyomas that are manifested clinically $[3,4]$.

Occasionally, leiomyomas can develop with uncommon growth patterns or in unusual locations, making their identification more challenging both clinically and radiologically. Diffuse peritoneal leiomyomatosis, intravenous leiomyomatosis, benign metastasizing leiomyomas, retroperitoneal leiomyomas, and parasitic leiomyomas are examples of leiomyomas with unusual growth patterns. Unusual sites of origin include the vulva, ovaries, urinary bladder, and urethra [2]. In this paper, we report one case of pelvic retroperitoneal leiomyoma associated to vulvar/ perineal leiomyomas.

\section{Case report}

A 47-year-old female patient was referred to our service due to a pelvic mass. She had a previous history of two open myomectomies (in 1995 and 1996), a cesarean section 
(in 1997), and a supracervical hysterectomy associated to the exeresis of a vulvar leiomyoma (in 2004). There was no evidence of disseminated intraperitoneal leiomyomatosis or myomas at any extrauterine location at the time of the last surgery. One year ago, she underwent a left salpingo-oophorectomy for a $9.2-\mathrm{cm}$ pelvic mass with the pathological finding of follicular ovarian cyst and tubal endometriosis.

She presented a 6-month history of deep dyspareunia, abdominal pain, dysuria, and pain during defecation. Her body mass index was $44.4 \mathrm{~kg} / \mathrm{m}^{2}$. On vaginal examination, there was a tender and firm mass at the vaginal vault and along the posterior vaginal wall. There was another tumor at the left vulvar/perineal region measuring around $6 \mathrm{~cm}$.

Transvaginal ultrasound showed a heterogeneous mass at the Douglas pouch measuring $11 \times 10 \times 9 \mathrm{~cm}$, with no atypical vascularization, and a heterogeneous, multilobulated, and well-delimited left perineal/vulvar mass measuring $6.3 \times 6.7 \mathrm{~cm}$ with no continuity to the pelvic mass. Magnetic resonance imaging demonstrated a multilobulated pelvic mass measuring $14 \times 10 \times 10 \mathrm{~cm}$ with large areas of fibrous remnants suggestive of uterine leiomyomatosis (Fig. 1). Based on these findings on imaging studies suggestive of benign leiomyoma, we decided to perform a laparoscopy with intraoperative frozen section.

Under general anesthesia, she underwent a laparoscopic surgery with the intraoperative finding of a retroperitoneal mass bulging the Douglas pouch and displacing the rectum to the right. All adhesions from the previous surgeries were released. The cervix was anteriorly retracted using a transparietal suspension to improve exposition. There was

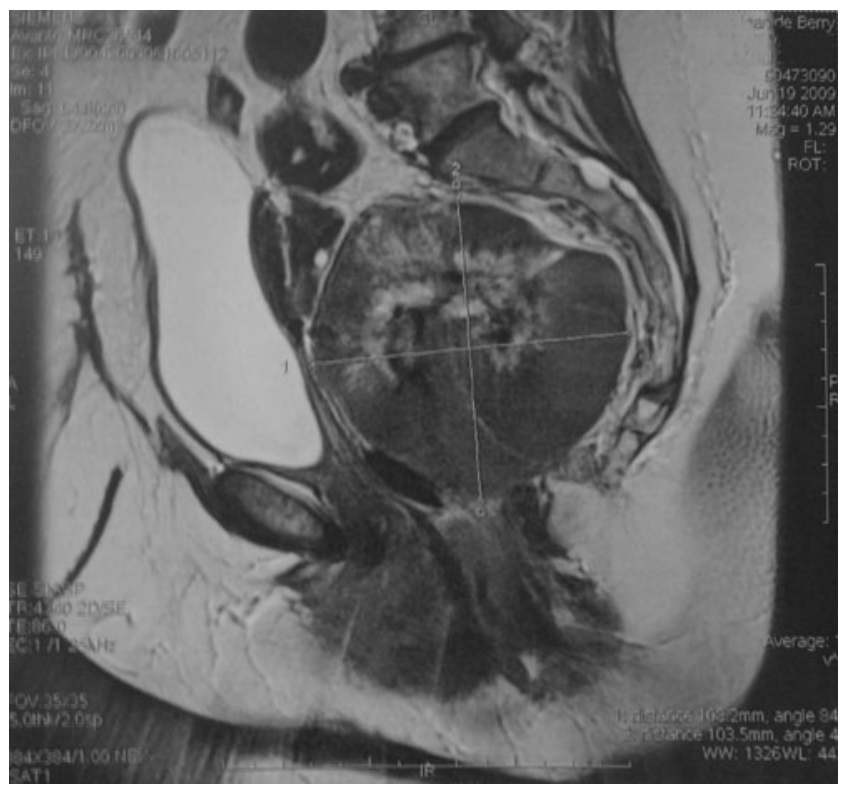

Fig. 1 Preoperative IRM demonstrating the presence of a pelvic tumor measuring $14 \times 10 \times 10 \mathrm{~cm}$ no direct communication between the residual uterine cervix and the mass. The retroperitoneum was opened over the mass (Figs. 2a and b) and a biopsy was performed for intraoperative frozen section which confirmed the preoperative diagnosis of leiomyoma. Careful dissection was conducted to free the tumor from adjacent structures (Fig. 2c), including posterior vaginal wall, pararectal fossa and levator ani muscules, rectum, and pevic sidewalls (Fig. 2d). After releasing the mass, it was morcellated to allow extraction from the abdominal cavity (Fig. 3). Right salpingo-oophorectomy completed the laparoscopic procedure. The second step of the surgery was the exeresis of the left vulvar/perineal tumor by vaginal approach. There were three leiomyomas measuring $0.5 \times 0.4,4 \times 2.5 \times 1$, and $4 \times$ $2.5 \times 2 \mathrm{~cm}$. Total weight of the pelvic mass retrieved after morcellation was $405 \mathrm{~g}$. Operative time was $210 \mathrm{~min}$. The difference between pre- and postoperative hemoglobin levels was $1.9 \mathrm{~g} / \mathrm{dl}$.

She presented an uneventful postoperative course and was discharged home 3 days after surgery.

Histopathology confirmed all masses to be leiomyomas. The retroperitoneal mass presented no areas of nuclear atypia or mitosis. There was extensive hyalinization and the residual smooth muscle fibers showed fibrinoid necrosis. The vulvar/perineal leiomyomas were well encapsulated and benign, and contained large areas of collagen deposits. Immunohistochemistry demonstrated the absence of hormonal receptors in all lesions. On postoperative day 30 , she was asymptomatic.

\section{Discussion}

Leiomyoma is more frequently found in the fourth and fifth decades of life [1]. Although the uterus is the most common site of origin of leiomyomas, the lesions arise as proliferations of smooth muscle cells, and they may develop at any site where such cells are found [2]. Rarely, extrauterine leiomyomas may be seen in the retroperitoneum $[1,2,5-$ 11] and along the labia majora [2, 12-14].

The etiology and pathogenesis of retroperitoneal leiomyomas are unclear. Leiomyomatous masses can be seen in the pelvic retroperitoneum in women with a concurrent uterine leiomyoma or a history of uterine leiomyoma. Rarely, the extrauterine masses may extend to the upper retroperitoneum, as high as the level of the renal hilum [2]. Several mechanisms of spread have been theorized on the basis of a spectrum of pathologic processes. There are some cases reported in patients previously submitted to hysterectomy $[5,6]$, and it might be speculated that retroperitoneal leiomyomas may be unrelated to uterine leiomyomas. Stutterecker et al. [5] proposed that retroperitoneal lesions could originate from embryonal remnants of müllerian or 
Fig. 2 a Opening the Douglas pouch retroperitoneum and visualization of the leiomyoma $(M)$ between the left uterosacral ligament $(L-U S L)$ and the rectum $(R)$. b Presence of a leiomyoma $(M)$ at the Douglas pouch between the left uterosacral ligament ( $L-U S L)$ and the rectum $(R)$; the rectum is displaced to the right, next to the right uterosacral ligament $(R$ $U S L)$. c Dissection of the leiomyoma $(M)$ from the adjacent structures. $L-U S L$ left uterosacral ligament, $R$-USL right uterosacral ligament, $C$ uterine cervix. d Final view after resection of the mass. $C$ uterine cervix, $L$-USL left uterosacral ligament, $R$-USL right uterosacral ligament, $R$ rectum
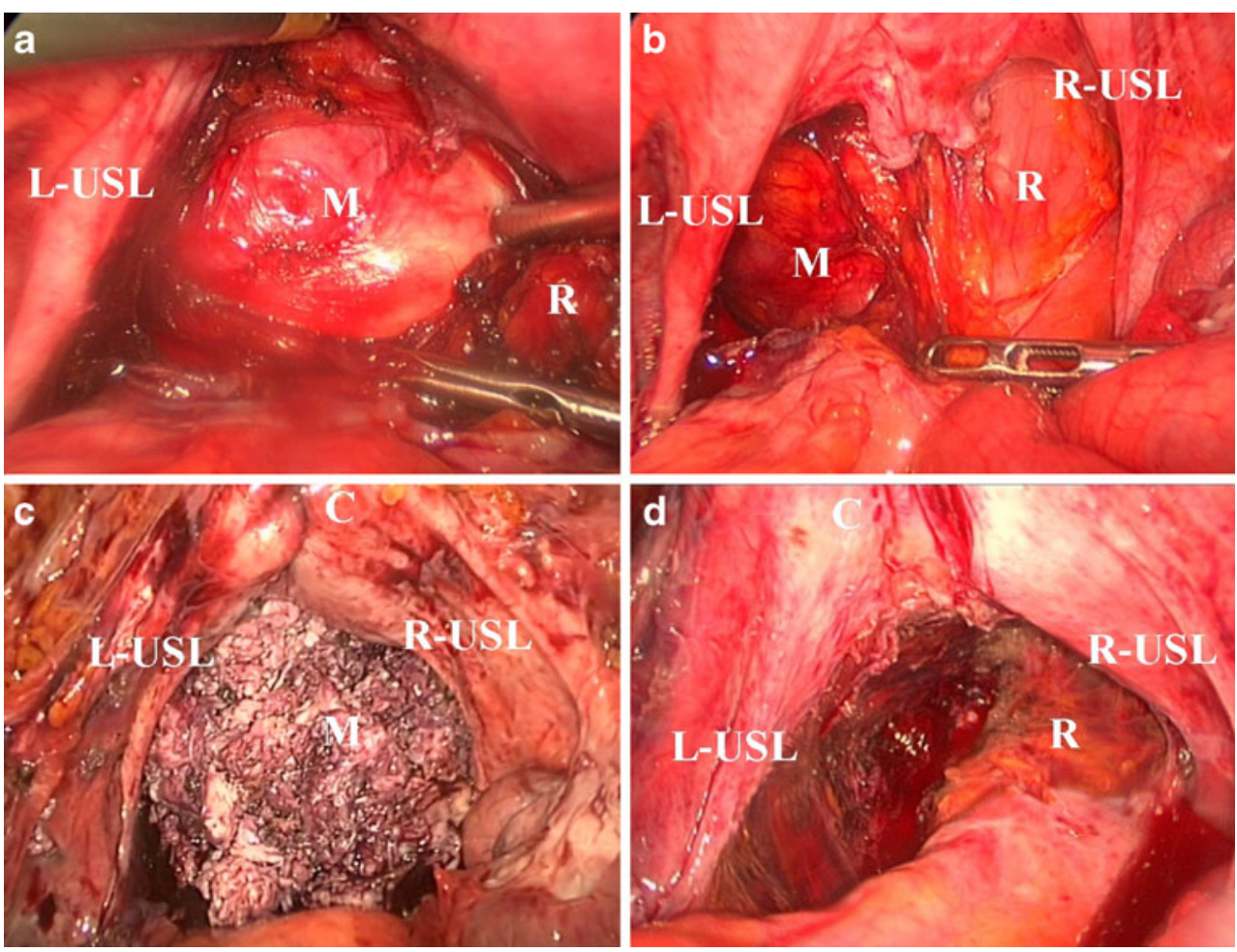

wolffian ducts. It also has been suggested that leiomyomas that are adherent to the broad ligament originate from hormonally sensitive smooth muscle elements of that ligament $[15,16]$. Occasionally, leiomyomas become adherent to surrounding structures (e.g., the broad ligament, omentum, or retroperitoneal connective tissue), develop an auxiliary blood supply, and lose their original attachment to the uterus, thus becoming "parasitic" [17, 18]. Classically, parasitic myomas occur spontaneously; however, part of the myometrium (or a small residual fibroid) can be left after vaginal [19] or abdominal hysterectomy and can receive blood supply, growing under stimulation by ovarian estrogen (residual parasitic theory) [9]. Alternatively, the

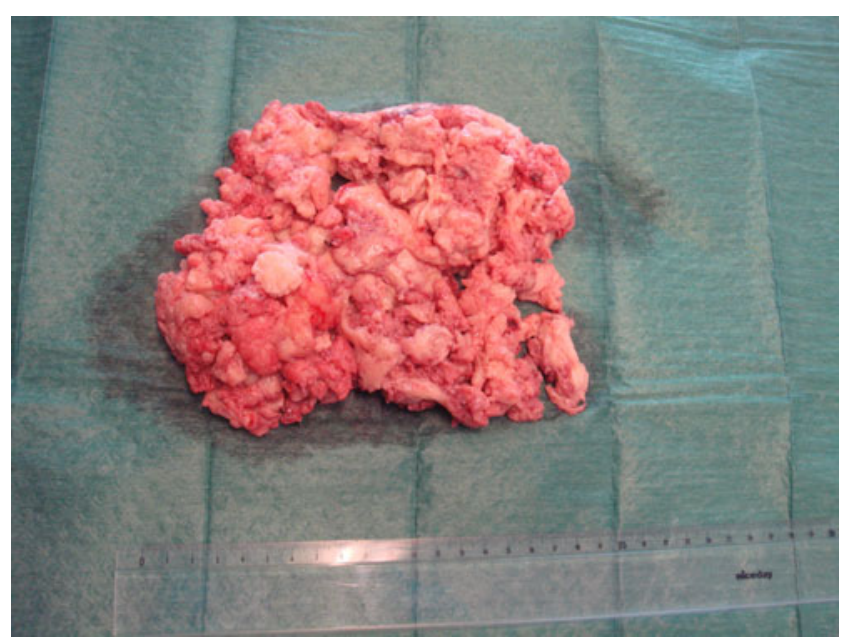

Fig. 3 Surgical specimen recent prevalence of minimally invasive endoscopic procedures $[20,21]$ may promote a new type of parasitic myoma that can be called an iatrogenic parasitic myoma [22, 23]. In this situation, the electric tissue morcellator produces minute fragments of myoma tissue and there is a possibility that these fragments will implant onto peritoneal or omental tissue [24] and grow. In addition, these retroperitoneal tumors can represent metastatic or synchronous primary lesions. Several hypotheses have been proposed to explain the metastasizing theory (the lungs are the most common site of metastatic involvement): (1) it could be a benign uterine tumor that spreads via a hematogenous route to the lungs or other organs [25], (2) it could be a surgically induced hematogenous spread, and the tumor would gain venous access from surgical trauma during hysterectomy [26], and (3) it could be a low-grade metastatic leiomyosarcoma [27]. Retroperitoneal tumors are sometimes seen on autopsy or are detected incidentally on routine checkup; so, they could also represent primary extrauterine leiomyomatosis unrelated to but coexisting with uterine leiomyomata [28]. In our patient, it is difficult to believe in the iatrogenic or the residual parasitic theories because the previous hysterectomy was supracervical. Therefore, the retroperitoneal space was not opened during the procedure. It is difficult to imagine that a piece of the myometrium left after morcellation infiltrated the retroperitoneum and developed there without any intraperitoneal lesion. Also, the metastasizing theory is not justified in our patient because neither pelvic lymph nodes nor blood vessels were involved in the retroperitoneal leiomyoma. 
We are not sure about the actual pathogenesis of this case, but maybe it could be a spontaneous parasitic leiomyoma arising from the residual cervix or a primary leiomyoma arising from embryonal remnants of müllerian or wolffian ducts.

More than $40 \%$ of patients affected by retroperitoneal leiomyomas have a concurrent uterine leiomyoma or a remote history of hysterectomy for treatment of a uterine leiomyoma. These tumors may enlarge considerably yet remain asymptomatic. Common symptoms include abdominal discomfort, fatigue, urinary symptoms, weight loss, and pelvic pain [11]. Rarely, they grow to become clinically apparent [1].

Although preoperative imaging and gross appearance are frequently typical for uterine leiomyomas, retroperitoneal leiomyomas are frequently misdiagnosed as malignant tumors [1] - tumors due to its unusual localization. Even though magnetic resonance imaging is the most reliable technique for evaluating retroperitoneal masses [2], the differential diagnosis between leiomyomas and leiomyosarcomas based on radiologic findings is difficult [29, 30]. Therefore, definitive diagnosis of a leiomyoma requires pathologic examination. US-guided percutaneous biopsy of the mass is helpful for determining its histologic composition preoperatively [2]. In some instances, determination of the exact nature of the retroperitoneal mass in a small histologic specimen might be difficult, and the true nature of the retroperitoneal mass can be only determined by complete histologic examination of the resected specimen $[1]$.

Despite the difficulties of approaching a pelvic retroperitoneal mass by laparoscopy, the performance of a minimally invasive surgery provides many advantages for the patient and is feasible and safe even for overweight/ obese women [31]. Compared with laparotomy, obese patients who undergo laparoscopy have reduced intraoperative bleeding, shorter hospitalization, less postoperative pain [32], and lower rates of postoperative wound infection [33]. Although most of previous reported cases of retroperitoneal leiomyomata were operated by laparotomy [1, 6-9], the laparoscopic approach is also feasible [34]. In our patient, the procedure was successfully performed by laparoscopy. The intraoperative frozen section was compatible with leiomyoma and the entire tumor could be dissected from the surrounding structures and removed. Reviewing 37 cases of retroperitoneal leiomyomata, Poliquin et al. [11] found that surgery was mostly curative with five reported cases of recurrence, three of which were then considered sarcomatous. In this setting, they recommend heightened surveillance after surgical treatment.

Differential diagnosis of retroperitoneal pelvic masses includes common benign and malignant neoplasms of neurogenic origin (schwannoma, paraganglioma, ganglio- neuroma, and extraadrenal pheochromocytoma) as well as teratoma, desmoid tumor, hemangioma, extraadrenal angiomyelolipoma, sarcoma, lymphoma, and metastatic tumors [35].

Our patient presented also vulvar/perineal leiomyomas which were resected by vaginal approach, because there was no sign of continuity with the retroperitoneal leiomyoma on preoperative pelvic magnetic resonance imaging (MRI). Fewer than 120 cases of smooth muscle tumors of the vulva have been reported in the literature [2]. They are thought to arise from smooth muscle cells within erectile tissue, blood vessel walls, or the round ligament [36]. The average tumor size varies from 0.5 to $15 \mathrm{~cm}$ [37], and findings at preoperative MRI may be suggestive of the benign nature of these tumors. The differential diagnosis includes benign and malignant entities such as Bartholin cysts, fibromas, lymphangiomas, soft-tissue sarcomas, and neurogenic tumors [2].

\section{Conclusions}

Pelvic retroperitoneal and vulvar leiomyomas are rare occurrences. In this paper, we reported one patient with concomitant retroperitoneal and vulvar lesions. Preoperative diagnosis based on MRI was suggestive of benign leiomyoma and laparoscopic approach for the retroperitoneal tumor was successfully performed. It is important to keep in mind the possibility of a retroperitoneal leiomyoma as a differential diagnosis of retroperitoneal masses in premenopausal women with previous history of hysterectomy for leiomyomas.

Conflict of interest There is no actual or potential conflict of interest in relation to this article.

\section{References}

1. Dursun P, Salman MC, Taskiran C, Yüce K, Ayhan A (2005) Retroperitoneal leiomyomatosis: a case report. Int J Gynecol Cancer 15:1222-1225

2. Fasih N, Prasad Shanbhogue AK, Macdonald DB, Fraser-Hill MA, Papadatos D, Kielar AZ et al (2008) Leiomyomas beyond the uterus: unusual locations, rare manifestations. Radiographics 28:1931-1948

3. Szklaruk J, Tamm EP, Choi H, Varavithya V (2003) MR imaging of common and uncommon large pelvic masses. Radiographics 23:403-424

4. Buttram VC Jr, Reiter RC (1981) Uterine leiomyomata: etiology, symptomatology, and management. Fertil Steril 36:433-445

5. Stutterecker D, Umek W, Tunn R, Sulzbacher I, Kainz C (2001) Leiomyoma in the space of Retzius: a report of 2 cases. Am J Obstet Gynecol 185:248-249

6. Maskery PJ (1970) Retroperitoneal leiomyomatosis following hysterectomy. Proc R Soc Med 63:55-56 
7. Sayer RA, Amundsen CL (2003) Giant pelvic retroperitoneal leiomyoma arising from the rectal wall. Obstet Gynecol 101:1132-1134

8. Kang WD, Kim SM, Kim YH, Choi HS (2009) Three isolated, large retroperitoneal leiomyomas. Arch Gynecol Obstet 280 (3):499-501

9. Fukamachi N, Togami S, Iwamoto I, Tsuji T, Nomoto M, Goto M et al (2007) Retroperitoneal uterine leiomyoma occurring 5 years after hysterectomy for fibroids. J Obstet Gynaecol Res 33:878881

10. Paal E, Miettinen M (2001) Retroperitoneal leiomyomas: a clinicopathologic and immunohistochemical study of 56 cases with a comparison to retroperitoneal leiomyosarcomas. Am J Surg Pathol 25:1355-1363

11. Poliquin V, Victory R, Vilos GA (2008) Epidemiology, presentation, and management of retroperitoneal leiomyomata: systematic literature review and case report. J Minim Invasive Gynecol $15: 152-160$

12. Reyad MM, Gazvani MR, Khine MM (2006) A rare case of primary leiomyoma of the vulva. J Obstet Gynaecol 26:73-74

13. Guven S, Esinler D, Salman MC, Gultekin M, Ayhan A (2005) Recurrent vulval leiomyoma in a postmenopausal patient mimicking vulval carcinoma. J Obstet Gynaecol 25:732-733

14. Pitukkijronnakorn S, Leelachaikul P, Chittacharoen A (2005) Labial leiomyoma: a case report. J Med Assoc Thai 88:118 119

15. Billings SD, Folpe AL, Weiss SW (2001) Do leiomyomas of deep soft tissue exist? An analysis of highly differentiated smooth muscle tumors of deep soft tissue supporting two distinct subtypes. Am J Surg Pathol 25:1134-1142

16. Rader JS, Binette SP, Brandt TD, Sreekanth S, Chhablani A (1990) Ileal hemorrhage caused by a parasitic uterine leiomyoma. Obstet Gynecol 76:531-534

17. Hendrickson MR, Kempson RL (1994) The uterine corpus. In: Hendrickson MR (ed) Diagnostic surgical pathology, 2nd edn. Raven Press, New York, pp 2091-2193

18. Brody S (1953) Parasitic fibroid. Am J Obstet Gynecol 65:13541356

19. Shukunami K, Nishijima K, Kurokawa T, Orisaka M, Yoshida Y, Kotsuji F (2006) A benign solitary uterine leiomyoma on the pelvic peritoneum detected long after the hysterectomy for fibroids. J Obstet Gynaecol 26:589

20. Istre O, Langebrekke A, Qvigstad E (2007) Changing hysterectomy technique from open abdominal to laparoscopic: new trend in Oslo, Norway. J Minim Invasive Gynecol 14:74-77

21. Hurst BS, Matthews ML, Marshburn PB (2005) Laparoscopic myomectomy for symptomatic uterine myomas. Fertil Steril 83:123

22. Paul PG, Koshy AK (2006) Multiple peritoneal parasitic myomas after laparoscopic myomectomy and morcellation. Fertil Steril $85: 492-493$
23. Sinha R, Sundaram M, Mahajan C, Sambhus A (2007) Multiple leiomyomas after laparoscopic hysterectomy: report of two cases. J Minim Invasive Gynecol 14:123-127

24. Takeda A, Mori M, Sakai K, Mitsui T, Nakamura H (2007) Parasitic peritoneal leiomyomatosis diagnosed 6 years after laparoscopic myomectomy with electric tissue morcellation: report of a case and review of the literature. J Minim Invasive Gynecol 14:770-775

25. Harper RS, Scully RE (1961) Intravenous leiomyomatosis of the uterus. A report of four cases. Obstet Gynecol 18:519-529

26. Wolff M, Silva F, Kaye G (1979) Pulmonary metastases (with admixed epithelial elements) from smooth muscle neoplasms. Report of nine cases, including three males. Am J Surg Pathol $3: 325-342$

27. Cho KR, Woodruff JD, Epstein JI (1989) Leiomyoma of the uterus with multiple extrauterine smooth muscle tumors: a case report suggesting multifocal origin. Hum Pathol 20:80-83

28. Goyle KK, Moore DF Jr, Garrett C, Goyle V (2003) Benign metastasizing leiomyomatosis: case report and review. Am J Clin Oncol 26:473-476

29. Schwartz LB, Zawin M, Carcangiu ML, Lange R, McCarthy S (1998) Does pelvic magnetic resonance imaging differentiate among the histologic subtypes of uterine leiomyomata? Fertil Steril 70:580-587

30. Arakawa A, Yasunaga T, Yano S, Morishita K, Nakashima K, Sato R et al (1993) Radiological findings of retroperitoneal leiomyoma and leiomyosarcoma: report of two cases. Comput Med Imaging Graph 17:125-131

31. O'Hanlan KA, Dibble SL, Fisher DT (2006) Total laparoscopic hysterectomy for uterine pathology: impact of body mass index on outcomes. Gynecol Oncol 103:938-941

32. Eltabbakh GH, Shamonki MI, Moody JM, Garafano LL (2000) Hysterectomy for obese women with endometrial cancer: laparoscopy or laparotomy? Gynecol Oncol 78:329-335

33. Obermair A, Manolitsas TP, Leung Y, Hammond IG, McCartney AJ (2005) Total laparoscopic hysterectomy versus total abdominal hysterectomy for obese women with endometrial cancer. Int $\mathrm{J}$ Gynecol Cancer 15:319-324

34. Hilger WS, Magrina JF (2006) Removal of pelvic leiomyomata and endometriosis five years after supracervical hysterectomy. Obstet Gynecol 108:772-774

35. Nishino M, Hayakawa K, Minami M, Yamamoto A, Ueda $H$, Takasu K (2003) Primary retroperitoneal neoplasms: CT and MR imaging findings with anatomic and pathologic diagnostic clues. Radiographics 23:45-57

36. Kaufman RH, Gardner HL (1965) Tumors of the vulva and vagina. Benign mesodermal tumors. Clin Obstet Gynecol 8:953-981

37. Nielsen GP, Rosenberg AE, Koerner FC, Young RH, Scully RE (1996) Smooth-muscle tumors of the vulva. A clinicopathological study of 25 cases and review of the literature. Am J Surg Pathol 20:779-793 